\title{
Rare hyperon decays with missing energy
}

\author{
Jusak Tandean \\ Department of Physics, National Taiwan University, \\ No. 1, Sec. 4, Roosevelt Rd., Taipei 106, Taiwan \\ Physics Division, National Center for Theoretical Sciences, \\ No. 101, Sec. 2, Kuang Fu Rd., Hsinchu 300, Taiwan \\ E-mail: jtandean@yahoo.com
}

ABSTRACT: We explore the strangeness-changing decays of the lightest hyperons into another baryon plus missing energy within and beyond the standard model (SM). In the SM these processes arise from the loop-induced quark transition $s \rightarrow d \nu \bar{\nu}$ and their branching fractions are estimated to be less than $10^{-11}$. In the presence of new physics (NP) the rates of these hyperon decays with missing energy could increase significantly with respect to the SM expectations because of modifications to the SM process or contributions from additional modes with new invisible particles. Adopting a model-independent approach and taking into account constraints from the kaon sector, we find that the current data on $K \rightarrow \pi \nu \bar{\nu}$ do not permit sizable NP impact on the hyperon decays via underlying operators having mainly parity-even quark parts. In contrast, NP operators with primarily parity-odd quark parts are much less restricted by the existing bounds on $K \rightarrow$ invisible and $K \rightarrow \pi \pi \nu \bar{\nu}$ and consequently could produce substantially amplifying effects on the hyperon modes. Their NP-enhanced branching fractions could reach levels potentially observable in the ongoing BESIII experiment.

Keywords: Beyond Standard Model, Effective Field Theories, Kaon Physics, Chiral Lagrangians

ARXIV EPRINT: 1901.10447 


\section{Contents}

1 Introduction 1

2 Interactions 2

3 Baryon decays 3

4 Kaon decays $\quad 6$

5 Numerical results $\quad 7$

$\begin{array}{lll}\text { 5.1 SM predictions and empirical information } & 7\end{array}$

5.2 Beyond SM 8

6 Conclusions 10

$\begin{array}{ll}\text { A Correspondences between quark and hadron transitions } & 11\end{array}$

B Additional kaon decay formulas 12

\section{Introduction}

The strangeness-changing quark transition $s \rightarrow d \mathbb{E}$, with missing energy $\mathbb{E}$ in the final state, is of great interest because it serves as an environment in which to test the standard model (SM) and therefore also to look for signals of possible new physics (NP) beyond it. Within the SM, this process is predominantly due to $s \rightarrow d \nu \bar{\nu}$ arising from $Z$-penguin and box diagrams with up-type quarks and the $W$ boson in the loops [1], the neutrino pair $(\nu \bar{\nu})$ being undetected. In the presence of NP, this SM contribution could be altered [2-9], and there might be invisible nonstandard states which are light enough and have $s d$ couplings to give rise to new $s \rightarrow d \notin$ channels [10-19].

Currently there are ongoing efforts to observe $s \rightarrow d \nu \bar{\nu}$ via the kaon decays $K^{+} \rightarrow \pi^{+} \nu \bar{\nu}$ and $K_{L} \rightarrow \pi^{0} \nu \bar{\nu}$ by the NA62 [20] and KOTO [21] Collaborations, respectively. These measurements might then probe for hints of $s \rightarrow d \notin$ beyond the SM as well. Additional kaon modes worth pursuing are $K_{L} \rightarrow \notin$ and $K \rightarrow \pi \pi^{\prime} \notin$, as only moderate bounds on $K \rightarrow \pi \pi^{\prime} \nu \bar{\nu}$ from direct searches are available [22]. Hence improved data on these extra modes would also be desirable. In the baryon sector, their counterparts are the strangenesschanging $(|\Delta S|=1)$ decays of light hyperons into another baryon plus missing energy, on which there is still no empirical information. Interestingly, measuring such processes in the BESIII experiment has recently been proposed and may be realized in the near future [7].

Here we have a look at these rare hyperon decays to investigate how much they may be affected by different NP possibilities, taking into account restrictions from the kaon sector. Initial studies on the hyperon modes due to new $s \rightarrow d \nu \bar{\nu}$ interactions with the same chiral 
structure as in the SM have been carried out in refs. [7, 9]. In this paper, we explore a more general scenario in which the underlying NP operators might involve other Lorentz structures and the invisible pair could be nonstandard fermions. It turns out that in this more general case the hyperon rates may be significantly enlarged with respect to their SM values and even reach potentially discoverable levels at BESIII.

As detailed later on in this analysis, such a possibility has to do with the fact that these kaon and hyperon decays do not probe the same portions of the underlying $s \rightarrow d \notin$ operators and with the kaon data situation at the moment. Particularly, $K \rightarrow \pi \notin$ is sensitive exclusively to the terms in the operators which have parity-even quark parts and $K \rightarrow \notin$ to the terms having parity-odd quark parts, whereas $K \rightarrow \pi \pi^{\prime} \notin$ and the hyperon modes can probe both. Given that the latest measurements of $K \rightarrow \pi \nu \bar{\nu}$ decays [20-22] have left only little room for NP to influence them, it follows that NP cannot raise the hyperon rates considerably above their SM expectations if it enters via $s \rightarrow d \notin$ operators with mainly parity-even quark parts. On the other hand, the constraints from the existing data on $K \rightarrow \mathbb{E}$ and $K \rightarrow \pi \pi^{\prime} \notin$ are relatively much weaker, implying that NP operators having primarily parity-odd quark parts are still allowed to yield sizable enhancing effects on the hyperon modes.

The organization of the paper is as follows. In section 2 we write down a number of effective low-energy operators contributing to $s \rightarrow d \notin$ which may be generated by NP. Without getting into model specifics, we treat the operators in a model-independent manner. In section 3 we first deal with the baryonic matrix elements pertinent to the corresponding hyperon decays with missing energy and subsequently derive their differential rates. Similarly, in section 4 we provide the formulas for the rare kaon decays of concern. In section 5 we present our numerical results. We begin by evaluating the SM predictions for the hyperon modes and comparing them to the proposed sensitivity reach of BESIII. Next we look at the kaon sector and examine its restraints on NP impacting the operators. We then show that some of the allowed NP couplings can amplify the hyperon rates to values that may be observable by BESIII. In section 6 , we give our conclusions. We collect extra formulas and further details in a couple of appendices.

\section{Interactions}

Beyond the SM, there could be new ingredients which induce modifications to the $s \rightarrow$ $d \nu \bar{\nu}$ transition in the SM and/or bring about additional $s \rightarrow d \notin$ channels with one or more invisible light nonstandard bosons or fermions emerging in the final states. These new particles could be stable or sufficiently long-lived to escape detection. Among such possibilities, in this study we focus on the $s \rightarrow d \notin$ scenario in which the missing energy is due to $f \bar{f}$ being emitted where $f$ is an electrically neutral, uncolored, and invisible Dirac fermion having spin $1 / 2$. Thus, $f$ could be the SM neutrino $\nu$, which is not detected, or a nonstandard fermion.

We consider $s d f \bar{f}$ interactions described by the low-energy effective Lagrangian

$$
\begin{aligned}
\mathcal{L}_{f}=-[ & \bar{d} \gamma^{\eta} s \bar{f} \gamma_{\eta}\left(\mathrm{C}_{f}^{\mathrm{V}}+\gamma_{5} \mathrm{C}_{f}^{\mathrm{A}}\right) f+\bar{d} \gamma^{\eta} \gamma_{5} s \bar{f} \gamma_{\eta}\left(\tilde{\mathrm{c}}_{f}^{\mathrm{V}}+\gamma_{5} \tilde{\mathrm{c}}_{f}^{\mathrm{A}}\right) f \\
& \left.+\bar{d} s \bar{f}\left(\mathrm{C}_{f}^{\mathrm{S}}+\gamma_{5} \mathrm{C}_{f}^{\mathrm{P}}\right) f+\bar{d} \gamma_{5} s \bar{f}\left(\tilde{\mathrm{c}}_{f}^{\mathrm{S}}+\gamma_{5} \tilde{\mathrm{c}}_{f}^{\mathrm{P}}\right) f\right]+ \text { H.c. }
\end{aligned}
$$


where in our model-independent approach $\mathrm{C}_{f}^{\mathrm{V}, \mathrm{A}, \mathrm{S}, \mathrm{P}}$ and $\tilde{\mathrm{C}}_{f}^{\mathrm{V}, \mathrm{A}, \mathrm{S}, \mathrm{P}}$ are free parameters which are generally complex and have the dimension of inverse squared mass. The terms in $\mathcal{L}_{f}$ are Lorentz invariant and respect the unbroken $\mathrm{SU}(3)_{\text {color }} \times \mathrm{U}(1)_{\mathrm{EM}}$ gauge symmetry. ${ }^{1}$ The grouping of the different operators in $\mathcal{L}_{f}$ according to the parity of their quark bilinears is convenient because in the decay rates to be examined the contributions of $\mathrm{C}_{f}^{\mathrm{V}, \mathrm{A}, \mathrm{S}, \mathrm{P}}$, belonging to $\mathcal{L}_{f}$ terms with parity-even quark bilinears, do not interfere with the contributions of $\tilde{\mathrm{C}}_{f}^{\mathrm{V}, \mathrm{A}, \mathrm{S}, \mathrm{P}}$, belonging to the terms with parity-odd quark bilinears.

As we will concentrate on exploring the potential implications of NP encoded in $\mathcal{L}_{f}$ for the transitions of light baryons and mesons at low hadronic energies, here we will not concern ourselves with how the parameters in $\mathcal{L}_{f}$ evolve from their high-energy values. It is nevertheless worth mentioning that for effective flavor-changing operators involving light quarks and SM-gauge-singlet dark particles the running of their coefficients from high to low energies has been estimated to be negligible [23].

In what follows we address how the NP may enlarge the rates of $|\Delta S|=1$ hyperon decays with missing energy compared to the SM expectations. Since $\mathcal{L}_{f}$ influences kaon decays as well, we need to ensure that the applied ranges of $\mathrm{C}_{f}^{\mathrm{V}, \mathrm{A}, \mathrm{S}, \mathrm{P}}$ and $\tilde{\mathrm{c}}_{f}^{\mathrm{V}, \mathrm{A}, \mathrm{S}, \mathrm{P}}$ are compatible with the available relevant data. In numerical work, we will assume the phenomenological viewpoint that these free parameters can have any values consistent with the empirical restrictions and perturbativity, and so some of them may be taken to be vanishing or much smaller than the others. This will allow us to look for parameter ranges that would translate into the maximal hyperon rates permitted by the kaon constraints. Although this may entail substantial differences among the NP parameters that appear rather unnatural, we optimistically suppose that models could be devised to accommodate them. ${ }^{2}$

\section{Baryon decays}

Our hyperon decays of interest are $\mathfrak{B} \rightarrow \mathfrak{B}^{\prime} f \bar{f}$ with $\mathfrak{B} \mathfrak{B}^{\prime}=\Lambda n, \Sigma^{+} p, \Xi^{0} \Lambda, \Xi^{0} \Sigma^{0}, \Xi^{-} \Sigma^{-}$, all the particles being spin- $1 / 2$ fermions, ${ }^{3}$ and $\Omega^{-} \rightarrow \Xi^{-} f \bar{f}$, where $\Omega^{-}$is a spin- $3 / 2$ hyperon. Some of these modes may be looked for in the near future at BESIII where, with one year's integrated luminosity, as many as $10^{6}-10^{8}$ hyperons $(\Lambda, \Sigma, \Xi$, and $\Omega)$ may be produced in $J / \psi$ and $\psi(2 S)$ decays [7].

To calculate the amplitudes for the hyperon decays, we need to know the baryonic matrix elements of the quark parts of the operators in eq. (2.1). We estimate the matrix elements with the aid of flavor-SU(3) chiral perturbation theory at leading order. Their derivation from the leading-order chiral Lagrangian is outlined in appendix A. We express

\footnotetext{
${ }^{1}$ For $f$ being a particle from a dark sector beyond the SM, the terms in $\mathcal{L}_{f}$ would constitute a subset of the independent operators detailed in [13], which include those containing dark particles of spin 0,1 , or $3 / 2$.

${ }^{2}$ For instance, a model possessing a heavy $Z^{\prime}$ boson which has family-nonuniversal purely-vector interactions with SM quarks [24] might be responsible for the $\bar{d} \gamma^{\eta} s$ terms in $\mathcal{L}_{f}$. If the $Z^{\prime}$ couplings to SM quarks are purely axial-vector instead [24-26], it might give rise to the $\bar{d} \gamma^{\eta} \gamma_{5} s$ terms.

${ }^{3}$ We do not include $\Sigma^{0} \rightarrow n f \bar{f}$ because its branching fraction is expected to be comparatively very suppressed due to the $\Sigma^{0}$ width being overwhelmingly dominated by the electromagnetic channel $\Sigma^{0} \rightarrow$ $\Lambda \gamma[22]$.
} 


\begin{tabular}{|c|c|c|c|c|c|}
\hline $\mathfrak{B}^{\prime} \mathfrak{B}$ & $n \Lambda$ & $p \Sigma^{+}$ & $\Lambda \Xi^{0}$ & $\Sigma^{0} \Xi^{0}$ & $\Sigma^{-} \Xi^{-}$ \\
\hline $\mathcal{V}_{\mathfrak{B}^{\prime} \mathfrak{B}}$ & $-\sqrt{\frac{3}{2}}$ & -1 & $\sqrt{\frac{3}{2}}$ & $\frac{-1}{\sqrt{2}}$ & 1 \\
\hline $\mathcal{A}_{\mathfrak{B}^{\prime} \mathfrak{B}}$ & $\frac{-1}{\sqrt{6}}(D+3 F)$ & $D-F$ & $\frac{-1}{\sqrt{6}}(D-3 F)$ & $\frac{-1}{\sqrt{2}}(D+F)$ & $D+F$ \\
\hline
\end{tabular}

Table 1. Values of $\mathcal{V}_{\mathfrak{B}^{\prime} \mathfrak{B}}$ and $\mathcal{A}_{\mathfrak{B}^{\prime} \mathfrak{B}}$ in eq. (3.1) for $\mathfrak{B} \mathfrak{B}^{\prime}=\Lambda n, \Sigma^{+} p, \Xi^{0} \Lambda, \Xi^{0} \Sigma^{0}, \Xi^{-} \Sigma^{-}$. The constants $D$ and $F$ come from the lowest-order chiral Lagrangian.

the results pertaining to $\mathfrak{B} \rightarrow \mathfrak{B}^{\prime} f \bar{f}$ as

$$
\begin{aligned}
\left\langle\mathfrak{B}^{\prime}\left|\bar{d} \gamma^{\eta} s\right| \mathfrak{B}\right\rangle & =\mathcal{V}_{\mathfrak{B}^{\prime} \mathfrak{B}} \bar{u}_{\mathfrak{B}^{\prime}} \gamma^{\eta} u_{\mathfrak{B}}, & \left\langle\mathfrak{B}^{\prime}\left|\bar{d} \gamma^{\eta} \gamma_{5} s\right| \mathfrak{B}\right\rangle & =\bar{u}_{\mathfrak{B}^{\prime}}\left(\gamma^{\eta} \mathcal{A}_{\mathfrak{B}^{\prime} \mathfrak{B}}-\frac{\mathcal{P}_{\mathfrak{B}^{\prime} \mathfrak{B}}}{B_{0}} \mathbb{Q}^{\eta}\right) \gamma_{5} u_{\mathfrak{B}}, \\
\left\langle\mathfrak{B}^{\prime}|\bar{d} s| \mathfrak{B}\right\rangle & =\mathcal{S}_{\mathfrak{B}^{\prime} \mathfrak{B}} \bar{u}_{\mathfrak{B}^{\prime}} u_{\mathfrak{B}}, & \left\langle\mathfrak{B}^{\prime}\left|\bar{d} \gamma_{5} s\right| \mathfrak{B}\right\rangle & =\mathcal{P}_{\mathfrak{B}^{\prime} \mathfrak{B}} \bar{u}_{\mathfrak{B}^{\prime}} \gamma_{5} u_{\mathfrak{B}},
\end{aligned}
$$

where $\mathcal{V}_{\mathfrak{B}^{\prime} \mathfrak{B}}$ and $\mathcal{A}_{\mathfrak{B}^{\prime} \mathfrak{B}}$ are constants whose values for the aforementioned $\mathfrak{B}^{\prime} \mathfrak{B}$ pairs are collected in table 1 , the $u$ s are Dirac spinors, $\mathbf{Q}=p_{\mathfrak{B}}-p_{\mathfrak{B}^{\prime}}$, with $p_{X}$ denoting the momentum of $X$,

$$
\mathcal{S}_{\mathfrak{B}^{\prime} \mathfrak{B}}=\frac{m_{\mathfrak{B}}-m_{\mathfrak{B}^{\prime}}}{m_{s}-\hat{m}} \mathcal{V}_{\mathfrak{B}^{\prime} \mathfrak{B}}, \quad \quad \mathcal{P}_{\mathfrak{B}^{\prime} \mathfrak{B}}=\mathcal{A}_{\mathfrak{B}^{\prime} \mathfrak{B}} B_{0} \frac{m_{\mathfrak{B}^{\prime}}+m_{\mathfrak{B}}}{m_{K}^{2}-Q^{2}},
$$

and the other quantities are defined in appendix A. For $\Omega^{-} \rightarrow \Xi^{-} f \bar{f}$ we have

$$
\begin{aligned}
\left\langle\Xi^{-}\left|\bar{d} \gamma^{\eta} s\right| \Omega^{-}\right\rangle & =0, & \left\langle\Xi^{-}\left|\bar{d} \gamma^{\eta} \gamma_{5} s\right| \Omega^{-}\right\rangle & =\mathcal{C} \bar{u}_{\Xi}\left(u_{\Omega}^{\eta}+\frac{\tilde{\mathrm{Q}}^{\eta} \tilde{\mathrm{Q}}_{\kappa}}{m_{K}^{2}-\tilde{\mathrm{Q}}^{2}} u_{\Omega}^{\kappa}\right), \\
\left\langle\Xi^{-}|\bar{d} s| \Omega^{-}\right\rangle & =0, & \left\langle\Xi^{-}\left|\bar{d} \gamma_{5} s\right| \Omega^{-}\right\rangle & =\frac{B_{0} \mathcal{C} \tilde{\mathrm{Q}}_{\kappa}}{\tilde{\mathrm{Q}}^{2}-m_{K}^{2}} \bar{u}_{\Xi} u_{\Omega}^{\kappa},
\end{aligned}
$$

where $\tilde{\mathrm{Q}}=p_{\Omega^{-}}-p_{\Xi^{-}}$and $u_{\Omega}^{\eta}$ is a Rarita-Schwinger spinor. Accordingly, in our approximation the amplitude for $\Omega^{-} \rightarrow \Xi^{-} f \bar{f}$, to be given below, does not contain the couplings $\mathrm{C}_{f}^{\mathrm{V}, \mathrm{A}, \mathrm{S}, \mathrm{P}}$. It is worth noting that the preceding baryonic matrix elements, and their mesonic counterparts to be discussed in section 4, fulfill the relations $\left\langle Y\left|\bar{d} \gamma_{\eta} s\right| X\right\rangle\left(p_{X}^{\eta}-p_{Y}^{\eta}\right)=$ $\left(m_{s}-\hat{m}\right)\langle Y|\bar{d} s| X\rangle$ and $\left\langle Y\left|\bar{d} \gamma_{\eta} \gamma_{5} s\right| X\right\rangle\left(p_{Y}^{\eta}-p_{X}^{\eta}\right)=\left(m_{s}+\hat{m}\right)\left\langle Y\left|\bar{d} \gamma_{5} s\right| X\right\rangle$ based on the free Dirac equation.

With eq. (3.1), we obtain the amplitude for $\mathfrak{B} \rightarrow \mathfrak{B}^{\prime} f \bar{f}$ to be

$$
\begin{aligned}
& \mathcal{M}_{\mathfrak{B} \rightarrow \mathfrak{B}^{\prime} f \bar{f}}=\bar{u}_{\mathfrak{B}^{\prime}} \gamma^{\eta} u_{\mathfrak{B}^{\prime}} \bar{u}_{f} \gamma_{\eta}\left(V_{\mathfrak{B B}^{\prime} f}+\gamma_{5} A_{\mathfrak{B B}^{\prime} f}\right) v_{\bar{f}} \\
& +\bar{u}_{\mathfrak{B}^{\prime}} \gamma^{\eta} \gamma_{5} u_{\mathfrak{B}} \bar{u}_{f} \gamma_{\eta}\left(\tilde{\mathrm{V}}_{\mathfrak{B B}^{\prime} f}+\gamma_{5} \tilde{\mathrm{A}}_{\mathfrak{B B}^{\prime} f}\right) v_{\bar{f}} \\
& +\bar{u}_{\mathfrak{B}^{\prime}} u_{\mathfrak{B}} \bar{u}_{f}\left(S_{\mathfrak{B B}^{\prime} f}+\gamma_{5} P_{\mathfrak{B B}^{\prime} f}\right) v_{\bar{f}} \\
& +\bar{u}_{\mathfrak{B}^{\prime}} \gamma_{5} u_{\mathfrak{B}} \bar{u}_{f}\left(\tilde{\mathrm{S}}_{\mathfrak{B B}^{\prime} f}+\gamma_{5} \tilde{\mathrm{P}}_{\mathfrak{B B}^{\prime} f}\right) v_{\bar{f}} \text {, }
\end{aligned}
$$

where the $v$ s are Dirac spinors for $\bar{f}$,

$$
\begin{array}{llll}
V_{\mathfrak{B B}^{\prime} f}=\mathcal{V}_{\mathfrak{B}^{\prime} \mathfrak{B}} C_{f}^{\mathrm{V}}, & A_{\mathfrak{B}^{\prime} f}=\mathcal{V}_{\mathfrak{B}^{\prime} \mathfrak{B}} \mathrm{C}_{f}^{\mathrm{A}}, & \tilde{\mathrm{V}}_{\mathfrak{B}^{\prime} f}=\mathcal{A}_{\mathfrak{B}^{\prime} \mathfrak{B}} \tilde{\mathrm{C}}_{f}^{\mathrm{V}}, & \tilde{\mathrm{A}}_{\mathfrak{B}^{\prime} f}=\mathcal{A}_{\mathfrak{B}^{\prime} \mathfrak{B}} \tilde{\mathrm{C}}_{f}^{\mathrm{A}}, \\
S_{\mathfrak{B B}^{\prime} f}=\mathcal{S}_{\mathfrak{B}^{\prime} \mathfrak{B}} \mathrm{C}_{f}^{\mathrm{S}}, & P_{\mathfrak{B B}^{\prime} f}=\mathcal{S}_{\mathfrak{B}^{\prime} \mathfrak{B}} \mathrm{C}_{f}^{\mathrm{P}}, & \tilde{\mathrm{S}}_{\mathfrak{B B}^{\prime} f}=\mathcal{P}_{\mathfrak{B}^{\prime} \mathfrak{B}} \tilde{\mathrm{C}}_{f}^{\mathrm{S}}, & \tilde{\mathrm{P}}_{\mathfrak{B B}^{\prime} f}=\mathcal{P}_{\mathfrak{B}^{\prime} \mathfrak{B}}\left(\tilde{\mathrm{C}}_{f}^{\mathrm{P}}-\frac{2 m_{f}}{B_{0}} \tilde{\mathrm{C}}_{f}^{\mathrm{A}}\right) .
\end{array}
$$


This leads to the differential decay rate

$$
\begin{aligned}
& \frac{d \Gamma_{\mathfrak{B} \rightarrow \mathfrak{B}^{\prime} f \bar{f}}}{d \hat{s}}=\frac{\beta \lambda_{\mathfrak{B}^{\prime}}^{1 / 2}}{128 \pi^{3} m_{\mathfrak{B}_{\mathfrak{B}}}^{3}}\left[\left(\beta^{2} \tilde{\sigma}_{+} \hat{s}+3 \tilde{\sigma}_{-} \hat{s}+\mathbb{F}\right)\left|V_{\mathfrak{B B}^{\prime} f}\right|^{2}+\left(\tilde{\sigma}_{+} \hat{s}+3 \beta^{2} \tilde{\sigma}_{-} \hat{s}+\mathbb{F}\right)\left|A_{\mathfrak{B B}^{\prime} f}\right|^{2}\right. \\
& +\left(\beta^{2} \tilde{\sigma}_{-} \hat{s}+3 \tilde{\sigma}_{+} \hat{s}+\mathbb{F}\right)\left|\tilde{\mathrm{V}}_{\mathfrak{B}_{\mathfrak{B}^{\prime} f}}\right|^{2}+\left(\tilde{\sigma}_{-} \hat{s}+3 \beta^{2} \tilde{\sigma}_{+} \hat{s}+\mathbb{F}\right)\left|\tilde{\mathrm{A}}_{\mathfrak{B}_{\mathfrak{B}^{\prime} f}}\right|^{2} \\
& +\tilde{\sigma}_{+}\left(\beta^{2}\left|S_{\mathfrak{B}_{\mathfrak{B}^{\prime} f}}\right|^{2}+\left|P_{\mathfrak{B}_{\mathfrak{B}^{\prime} f}}\right|^{2}\right) \hat{s}+\tilde{\sigma}_{-}\left(\beta^{2}\left|\tilde{\mathrm{S}}_{\mathfrak{B B}^{\prime} f}\right|^{2}+\left|\tilde{\mathrm{P}}_{\mathfrak{B}_{\mathfrak{B}^{\prime} f}}\right|^{2}\right) \hat{s} \\
& \left.+4 m_{f} \operatorname{Re}\left(\tilde{\sigma}_{+} \mathrm{M}_{-} A_{\mathfrak{B}_{\mathfrak{B}^{\prime} f}}^{*} P_{\mathfrak{B B}^{\prime} f}-\tilde{\sigma}_{-} \mathrm{M}_{+} \tilde{\mathrm{A}}_{\mathfrak{B}_{\mathfrak{B}^{\prime} f}}^{*} \tilde{\mathrm{P}}_{\mathfrak{B B}^{\prime} f}\right)\right],
\end{aligned}
$$

where

$$
\begin{aligned}
& \beta=\sqrt{1-\frac{4 m_{f}^{2}}{\hat{s}}}, \quad \hat{s}=\left(p_{f}+p_{\bar{f}}\right)^{2}, \quad \lambda_{X Y}=m_{X}^{4}-2\left(m_{Y}^{2}+\hat{s}\right) m_{X}^{2}+\left(m_{Y}^{2}-\hat{s}\right)^{2}, \\
& \tilde{\sigma}_{ \pm}=\mathrm{M}_{ \pm}^{2}-\hat{s}, \quad \mathrm{M}_{ \pm}=m_{\mathfrak{B}} \pm m_{\mathfrak{B}^{\prime}}, \quad \mathbb{F}=\frac{3-\beta^{2}}{3} \lambda_{\mathfrak{B}_{\mathfrak{B}^{\prime}}}+2 \beta^{2}\left(\hat{s}-m_{\mathfrak{B}^{\prime}}^{2}-m_{\mathfrak{B}^{\prime}}^{2}\right) \hat{s}
\end{aligned}
$$

The rate results from integrating the differential rate over $4 m_{f}^{2} \leq \hat{s} \leq\left(m_{\mathfrak{B}}-m_{\mathfrak{B}^{\prime}}\right)^{2}$. In eq. (3.6) we observe that the $\mathrm{C}_{f}^{\mathrm{V}, \mathrm{A}, \mathrm{S}, \mathrm{P}}$ terms do not interfere with the $\tilde{\mathrm{c}}_{f}^{\mathrm{V}, \mathrm{A}, \mathrm{S}, \mathrm{P}}$ ones, which is also the case in the kaon decays to be examined later on.

For the $\Omega^{-}$decay we find

$$
\mathcal{M}_{\Omega^{-} \rightarrow \Xi^{-} f \bar{f}}=\mathcal{C}\left(g_{\kappa \varsigma}+\frac{\tilde{\mathrm{Q}}_{\kappa} \tilde{\mathrm{Q}}_{\varsigma}}{m_{K^{0}}^{2}-\hat{s}}\right) \bar{u}_{\Xi} u_{\Omega}^{\kappa} \bar{u}_{f}\left[\gamma^{\varsigma}\left(\tilde{\mathrm{c}}_{f}^{\mathrm{V}}+\gamma_{5} \tilde{\mathrm{c}}_{f}^{\mathrm{A}}\right)-\frac{B_{0} \tilde{\mathrm{Q}}^{\varsigma}}{m_{K^{0}}^{2}}\left(\tilde{\mathrm{c}}_{f}^{\mathrm{S}}+\gamma_{5} \tilde{\mathrm{c}}_{f}^{\mathrm{P}}\right)\right] v_{\bar{f}},
$$

where $\tilde{\mathrm{Q}}=p_{\Omega^{-}}-p_{\Xi^{-}}$. The resulting differential rate is

$$
\begin{aligned}
\frac{d \Gamma_{\Omega^{-} \rightarrow \Xi^{-} f \bar{f}}}{d \hat{s}}=\frac{\beta \lambda_{\Omega^{-} \Xi^{-}}^{1 / 2} \mathcal{C}^{2} \hat{s}}{1536 \pi^{3} m_{\Omega^{-}}^{5}} & \left\{\left(3-\beta^{2}\right) \mathcal{G}\left|\tilde{\mathrm{c}}_{f}^{\mathrm{V}}\right|^{2}+\left[2 \beta^{2} \mathcal{G}+\left(1-\beta^{2}\right) \tilde{K} m_{K^{0}}^{4}\right]\left|\tilde{\mathrm{c}}_{f}^{\mathrm{A}}\right|^{2}\right. \\
& \left.+B_{0}^{2}\left(\beta^{2}\left|\tilde{\mathrm{c}}_{f}^{\mathrm{S}}\right|^{2}+\left|\tilde{\mathrm{c}}_{f}^{\mathrm{P}}\right|^{2}\right) \tilde{K} \hat{s}-4 B_{0} \tilde{K} m_{f} m_{K^{0}}^{2} \operatorname{Re}\left(\tilde{\boldsymbol{c}}_{f}^{\mathrm{A} * \tilde{c}_{f}^{\mathrm{P}}}\right)\right\}
\end{aligned}
$$

where

$$
\mathcal{G}=\left(\frac{\lambda_{\Omega^{-} \Xi^{-}}}{3}+4 m_{\Omega^{-}}^{2} \hat{s}\right) \frac{\left(m_{\Omega^{-}}+m_{\Xi^{-}}\right)^{2}-\hat{s}}{\hat{s}}, \quad \tilde{K}=\lambda_{\Omega^{-} \Xi^{-}} \frac{\left(m_{\Omega^{-}}+m_{\Xi^{-}}\right)^{2}-\hat{s}}{\left(m_{K^{0}}^{2}-\hat{s}\right)^{2} \hat{s}} .
$$

In eq. (3.1) there are form-factor effects not yet taken into account. To incorporate them, in numerical work we modify $\mathcal{V}_{\mathfrak{B}^{\prime} \mathfrak{B}}$ and $\mathcal{A}_{\mathfrak{B}^{\prime} \mathfrak{B}}$ to $\left(1+2 Q^{2} / M_{V}^{2}\right) \mathcal{V}_{\mathfrak{B}^{\prime} \mathfrak{B}}$ and $\left(1+2 Q^{2} / M_{A}^{2}\right) \mathcal{A}_{\mathfrak{B}^{\prime} \mathfrak{B}}$, respectively, with $M_{V}=0.97 \mathrm{GeV}$ and $M_{A}=1.25 \mathrm{GeV}$, following the commonly used parametrization in experimental analyses of hyperon semileptonic decays [27-30] and assuming isospin symmetry. Analogously, since $\tilde{\mathrm{Q}}^{2}$ in $\Omega^{-} \rightarrow \Xi^{-} f \bar{f}$ has a significantly wider range than $Q^{2}$ in $\mathfrak{B} \rightarrow \mathfrak{B}^{\prime} f \bar{f}$, in the $\Omega^{-}$decay rate we implement the change $\mathcal{C} \rightarrow \mathcal{C} /\left(1-\tilde{\mathrm{Q}}^{2} / M_{A}^{2}\right)^{2}$. These modifications turn out to translate into increases of the rates by up to $\sim 16$ percent. 


\section{Kaon decays}

For $K_{L, S} \rightarrow f \bar{f}$ the relevant hadronic matrix elements are

$$
\left\langle 0\left|\bar{d} \gamma^{\eta} \gamma_{5} s\right| \bar{K}^{0}\right\rangle=\left\langle 0\left|\bar{s} \gamma^{\eta} \gamma_{5} d\right| K^{0}\right\rangle=-i f_{K} p_{K}^{\eta}, \quad\left\langle 0\left|\bar{d} \gamma_{5} s\right| \bar{K}^{0}\right\rangle=\left\langle 0\left|\bar{s} \gamma_{5} d\right| K^{0}\right\rangle=i B_{0} f_{K},
$$

with $f_{K}=155.6(4) \mathrm{MeV}$ [22] being the kaon decay constant, while for $K \rightarrow \pi f \bar{f}$

$$
\begin{aligned}
\left\langle\pi^{-}\left|\bar{d} \gamma^{\eta} s\right| K^{-}\right\rangle & =-\left\langle\pi^{+}\left|\bar{s} \gamma^{\eta} d\right| K^{+}\right\rangle=\left(p_{K}^{\eta}+p_{\pi}^{\eta}\right) f_{+}+\left(f_{0}-f_{+}\right) q_{K \pi}^{\eta} \frac{m_{K}^{2}-m_{\pi}^{2}}{q_{K \pi}^{2}}, \\
\left\langle\pi^{-}|\bar{d} s| K^{-}\right\rangle & =+\left\langle\pi^{+}|\bar{s} d| K^{+}\right\rangle=B_{0} f_{0}, \quad q_{K \pi}=p_{K}-p_{\pi},
\end{aligned}
$$

where $f_{+}$and $f_{0}$ represent form factors which are functions of $q_{K \pi}^{2}$. In addition, assuming isospin symmetry, we have $\left\langle\pi^{0}\left|\bar{d}\left(\gamma^{\eta}, 1\right) s\right| \bar{K}^{0}\right\rangle=\left\langle\pi^{0}\left|\bar{s}\left(-\gamma^{\eta}, 1\right) d\right| K^{0}\right\rangle=$ $-\left\langle\pi^{-}\left|\bar{d}\left(\gamma^{\eta}, 1\right) s\right| K^{-}\right\rangle / \sqrt{2}$ and also $\left\langle\pi^{-}\left|\bar{d} \gamma^{\eta} s\right| K^{-}\right\rangle=\left\langle\pi^{+}\left|\bar{u} \gamma^{\eta} s\right| \bar{K}^{0}\right\rangle$. We can then adopt $f_{+, 0}=\mathrm{f}_{+}(0)\left(1+\lambda_{+, 0} q_{K \pi}^{2} / m_{\pi^{+}}^{2}\right)$ with $\lambda_{+}=0.0271(10)$ and $\lambda_{0}=0.0142(23)$ from $K_{L} \rightarrow \pi^{+} \mu^{-} \nu$ measurements [22] as well as $\mathrm{f}_{+}(0)=0.9681(23)$ from lattice computations [31]. For $K^{-} \rightarrow \pi^{0} \pi^{-} f \bar{f}$ and $K_{L} \rightarrow \pi^{0} \pi^{0} f \bar{f}$, from the results in appendix A we obtain

$$
\begin{aligned}
\left\langle\pi^{0}\left(p_{0}\right) \pi^{-}\left(p_{-}\right)\left|\bar{d}\left(\gamma^{\eta}, 1\right) \gamma_{5} s\right| K^{-}\right\rangle & =\frac{i \sqrt{2}}{f_{K}}\left[\left(p_{0}^{\eta}-p_{-}^{\eta}, 0\right)+\frac{\left(p_{0}-p_{-}\right) \cdot \tilde{q}}{m_{K}^{2}-\tilde{q}^{2}}\left(\tilde{q}^{\eta},-B_{0}\right)\right], \\
\left\langle\pi^{0}\left(p_{1}\right) \pi^{0}\left(p_{2}\right)\left|\bar{d}\left(\gamma^{\eta}, 1\right) \gamma_{5}\right| \bar{K}^{0}\right\rangle & =\frac{i}{f_{K}}\left[\left(p_{1}^{\eta}+p_{2}^{\eta}, 0\right)+\frac{\left(p_{1}+p_{2}\right) \cdot \tilde{q}}{m_{K}^{2}-\tilde{q}^{2}}\left(\tilde{q}^{\eta},-B_{0}\right)\right],
\end{aligned}
$$

where $\tilde{q}=p_{K^{-}}-p_{0}-p_{-}=p_{\bar{K}^{0}}-p_{1}-p_{2}$. In the $K^{-}$case, there is additionally a small contribution involving one of the parity-even quark transitions, $\left\langle\pi^{0} \pi^{-}\left|\bar{d} \gamma^{\eta} s\right| K^{-}\right\rangle \neq 0$, which arises from the anomaly Lagrangian [13], at next-to-leading order in the chiral expansion, and which we have therefore neglected. Since the existing empirical limits on $K \rightarrow \pi \pi^{\prime} f \bar{f}$ are not very stringent, we also ignore form-factor effects in calculating their rates.

It follows that the amplitudes for $K_{L} \rightarrow f \bar{f}$ and $K_{S} \rightarrow f \bar{f}$ induced by $\mathcal{L}_{f}$ are

$$
\mathcal{M}_{K_{L, S} \rightarrow f \bar{f}}=i \bar{u}_{f}\left(S_{K_{L, S} f}+\gamma_{5} P_{K_{L, S} f}\right) v_{\bar{f}},
$$

leading to the decay rates

$$
\Gamma_{K_{L, S} \rightarrow f \bar{f}}=\frac{m_{K^{0}}}{8 \pi}\left(\tilde{\beta}^{3}\left|S_{K_{L, S} f}\right|^{2}+\tilde{\beta}\left|P_{K_{L, S} f}\right|^{2}\right),
$$

where $\tilde{\beta}=\left(1-4 m_{f}^{2} / m_{K^{0}}^{2}\right)^{1 / 2}$,

$$
\begin{array}{ll}
S_{K_{L} f}=i \sqrt{2} B_{0} f_{K} \operatorname{Im} \tilde{\mathrm{c}}_{f}^{\mathrm{S}}, & P_{K_{L} f}=-\sqrt{2} f_{K} \operatorname{Re}\left(2 m_{f} \tilde{\mathrm{c}}_{f}^{\mathrm{A}}-B_{0} \tilde{\mathrm{c}}_{f}^{\mathrm{P}}\right), \\
S_{K_{S} f}=-\sqrt{2} B_{0} f_{K} \operatorname{Re} \tilde{\mathrm{c}}_{f}^{\mathrm{S}}, & P_{K_{S} f}=i \sqrt{2} f_{K} \operatorname{Im}\left(2 m_{f} \tilde{\mathrm{c}}_{f}^{\mathrm{A}}-B_{0} \tilde{\mathrm{c}}_{f}^{\mathrm{P}}\right) .
\end{array}
$$

Thus, $K_{L, S} \rightarrow f \bar{f}$ are not sensitive to $\mathrm{C}_{f}^{\mathrm{V}, \mathrm{A}, \mathrm{S}, \mathrm{P}}$ and $\tilde{\mathrm{c}}_{f}^{\mathrm{V}}$.

The amplitude for $K \rightarrow \pi f \bar{f}$ has the form

$$
\mathcal{M}_{K \rightarrow \pi f \bar{f}}=\bar{u}_{f}\left(S_{K \pi f}+P_{K \pi f} \gamma_{5}\right) v_{\bar{f}} .
$$


We put the resulting differential rates of $K^{-} \rightarrow \pi^{-} f \bar{f}$ and $K_{L, S} \rightarrow \pi^{0} f \bar{f}$ in appendix B, which also shows that these modes, in contrast to $K_{L, S} \rightarrow f \bar{f}$, can probe $\mathrm{C}_{f}^{\mathrm{V}, \mathrm{A}, \mathrm{S}, \mathrm{P}}$, but $\operatorname{not} \tilde{c}_{f}^{\mathrm{V}, \mathrm{A}, \mathrm{S}, \mathrm{P}}$.

For $K^{-} \rightarrow \pi^{0} \pi^{-} f \bar{f}$ and $K_{L} \rightarrow \pi^{0} \pi^{0} f \bar{f}$, we get

$$
\begin{aligned}
\mathcal{M}_{K^{-} \rightarrow \pi^{0} \pi^{-} f \bar{f}}=\frac{i \sqrt{2}}{f_{K}} \bar{u}_{f}\{ & {\left[2 \gamma_{5} \tilde{\mathrm{c}}_{f}^{\mathrm{A}} m_{f}-B_{0}\left(\tilde{\mathrm{c}}_{f}^{\mathrm{S}}+\gamma_{5} \tilde{\mathrm{c}}_{f}^{\mathrm{P}}\right)\right] \frac{p_{K} \cdot\left(p_{0}-p_{-}\right)}{m_{K}^{2}-\hat{s}} } \\
\left.+\left(\not p_{0}-\not p_{-}\right)\left(\tilde{\mathrm{c}}_{f}^{\mathrm{V}}+\gamma_{5} \tilde{\mathrm{c}}_{f}^{\mathrm{A}}\right)\right\} v_{\bar{f}}, & \\
\mathcal{M}_{K_{L} \rightarrow \pi^{0} \pi^{0} f \bar{f}}=\frac{i \sqrt{2}}{f_{K}} \bar{u}_{f}\{ & {\left[2 \gamma_{5} \operatorname{Re} \tilde{\mathrm{c}}_{f}^{\mathrm{A}} m_{f}-B_{0}\left(i \operatorname{Im} \tilde{\mathrm{c}}_{f}^{\mathrm{S}}+\gamma_{5} \operatorname{Re} \tilde{\mathrm{c}}_{f}^{\mathrm{P}}\right)\right] \frac{p_{K} \cdot\left(p_{f}+p_{\bar{f}}\right)-\hat{s}}{m_{K}^{2}-\hat{s}} } \\
& \left.+\left(\not p_{1}+\not p_{2}\right)\left(\operatorname{Re} \tilde{\mathrm{c}}_{f}^{\mathrm{V}}+\gamma_{5} \operatorname{Re} \tilde{\mathrm{c}}_{f}^{\mathrm{A}}\right)\right\} v_{\bar{f}} .
\end{aligned}
$$

Their differential rates are also relegated to appendix B.

We notice from eqs. (3.5) and (3.6) that, unlike these kaon modes, $\mathfrak{B} \rightarrow \mathfrak{B}^{\prime} f \bar{f}$ are sensitive to both $\mathrm{C}_{f}^{\mathrm{V}, \mathrm{A}, \mathrm{S}, \mathrm{P}}$ and $\tilde{\mathrm{C}}_{f}^{\mathrm{V}, \mathrm{A}, \mathrm{S}, \mathrm{P}}$. It is therefore advantageous to measure $\mathfrak{B} \rightarrow \mathfrak{B}^{\prime} \mathbb{E}$, as the acquired data could supply information on $s \rightarrow d \notin$ which is complementary to that from the kaon sector.

\section{Numerical results}

\subsection{SM predictions and empirical information}

Within the SM, our hyperon decays of interest are induced by effective short-distance $s d \nu_{l} \bar{\nu}_{l}$ interactions, with $l=e, \mu, \tau$, described by [1]

$$
\mathcal{L}_{s d \nu \nu}^{\mathrm{SM}}=\frac{-\alpha_{\mathrm{e}} G_{\mathrm{F}}}{\sqrt{8} \pi s_{\mathrm{W}}^{2}} \sum_{l=e, \mu, \tau}\left(V_{t d}^{*} V_{t s} X_{t}+V_{c d}^{*} V_{c s} X_{c}^{l}\right) \bar{d} \gamma^{\eta}\left(1-\gamma_{5}\right) s \overline{\nu_{l}} \gamma_{\eta}\left(1-\gamma_{5}\right) \nu_{l}+\text { H.c. }
$$

where $\alpha_{\mathrm{e}} \simeq 1 / 128$ and $G_{\mathrm{F}}$ are the usual fine-structure and Fermi constants, $s_{\mathrm{W}}^{2} \equiv \sin ^{2} \theta_{\mathrm{W}}=$ 0.231 with $\theta_{\mathrm{W}}$ being the Weinberg angle, $V_{q q^{\prime}}$ are Cabibbo-Kobayashi-Maskawa (CKM) matrix elements, $X_{t}=1.481(9)$ comes from $t$-quark loops [32], and $X_{c}^{e}=X_{c}^{\mu} \simeq 1.0 \times$ $10^{-3}$ and $X_{c}^{\tau} \simeq 7 \times 10^{-4}$ are $c$-quark contributions [1]. To evaluate $\mathcal{B}\left(\mathfrak{B} \rightarrow \mathfrak{B}^{\prime} \nu \bar{\nu}\right)_{\mathrm{SM}}=$ $\sum_{l} \mathcal{B}\left(\mathfrak{B} \rightarrow \mathfrak{B}^{\prime} \nu_{l} \bar{\nu}_{l}\right)_{\mathrm{SM}}$ we can apply eq. (3.6) with $\mathrm{C}_{\nu_{l}}^{\mathrm{V}}=-\mathrm{C}_{\nu_{l}}^{\mathrm{A}}=-\tilde{\mathrm{c}}_{\nu_{l}}^{\mathrm{V}}=\tilde{\mathrm{c}}_{\nu_{l}}^{\mathrm{A}}=\alpha_{\mathrm{e}} G_{\mathrm{F}}\left(\lambda_{t} X_{t}+\right.$ $\left.\lambda_{c} X_{c}^{l}\right) /\left(\sqrt{8} \pi s_{\mathrm{W}}^{2}\right)$ and $\mathrm{C}_{\nu_{l}}^{\mathrm{S}, \mathrm{P}}=\tilde{\mathrm{c}}_{\nu_{l}}^{\mathrm{S}, \mathrm{P}}=0$. Similarly, we can determine $\mathcal{B}\left(\Omega^{-} \rightarrow \Xi^{-} \nu \bar{\nu}\right)_{\mathrm{SM}}$ using eq. (3.9). ${ }^{4}$ Thus, with the central values of the input parameters, we arrive at the entries in the second row of table $2 .{ }^{5}$ The CKM factors and $X_{t, c}$ contribute an uncertainty of almost $10 \%$ to the SM predictions, the estimation of the baryonic matrix elements has an uncertainty of $\sim 20 \%$, and so the total uncertainty of the branching-fraction predictions is about $50 \%$.

\footnotetext{
${ }^{4}$ These decays also receive long-distance contributions mediated by the $Z$ boson, such as $\mathfrak{B} \rightarrow \mathfrak{B}^{\prime} Z^{*} \rightarrow$ $\mathfrak{B}^{\prime} \nu \bar{\nu}$, but their size is estimated to be small compared to the short-distance SM contribution.

${ }^{5}$ Our numbers are roughly comparable to the ones given in [9], but therein the flavor-SU(3) properties of the baryon interactions were not taken into account and different momentum-dependences of the baryonic matrix elements were used.
} 


\begin{tabular}{|c|c|c|c|c|c|c|}
\hline Decay mode & $\Lambda \rightarrow n \nu \bar{\nu}$ & $\Sigma^{+} \rightarrow p \nu \bar{\nu}$ & $\Xi^{0} \rightarrow \Lambda \nu \bar{\nu}$ & $\Xi^{0} \rightarrow \Sigma^{0} \nu \bar{\nu}$ & $\Xi^{-} \rightarrow \Sigma^{-} \nu \bar{\nu}$ & $\Omega^{-} \rightarrow \Xi^{-} \nu \bar{\nu}$ \\
\hline $\begin{array}{c}\text { SM branching } \\
\text { fraction }\end{array}$ & $7.1 \times 10^{-13}$ & $4.3 \times 10^{-13}$ & $6.3 \times 10^{-13}$ & $1.0 \times 10^{-13}$ & $1.3 \times 10^{-13}$ & $4.9 \times 10^{-12}$ \\
\hline $\begin{array}{c}\text { Expected BESIII } \\
\text { sensitivity [7] }\end{array}$ & $3 \times 10^{-7}$ & $4 \times 10^{-7}$ & $8 \times 10^{-7}$ & $9 \times 10^{-7}$ & - & $2.6 \times 10^{-5}$ \\
\hline
\end{tabular}

Table 2. The branching fractions of $|\Delta S|=1$ hyperon decays with missing energy in the SM and the corresponding expected sensitivities of BESIII [7].

At present there are no data available on these hyperon transitions, but this situation may change in the near future if BESIII performs a quest for them. In the last row of table 2 we quote its estimated sensitivities [90\% confidence level (CL)] for their branching fractions [7]. Clearly, it is unlikely that the SM predictions will be tested anytime soon. Nevertheless, as we demonstrate in the next section, it is possible for NP to amplify the branching fractions to levels potentially reachable by BESIII.

Turning to the kaon sector, we see that eqs. (4.5) and (4.6) imply $\Gamma_{K_{L, S} \rightarrow \nu \bar{\nu}}^{\mathrm{SM}}=0$ due to the neutrinos' masslessness in the SM. If it is supplemented with nonzero neutrino masses, their highest one from the direct limit $m_{\nu_{\tau}}^{\exp }<18.2 \mathrm{MeV}[22]$ translates into the maximal values $\mathcal{B}\left(K_{L} \rightarrow \nu \bar{\nu}\right)_{\mathrm{SM}} \simeq 1 \times 10^{-10}$ and $\mathcal{B}\left(K_{S} \rightarrow \nu \bar{\nu}\right)_{\mathrm{SM}} \simeq 2 \times 10^{-14}$. Therefore, observations of $\mathcal{B}\left(K_{L, S} \rightarrow \not{E}\right) \gg 10^{-10}$ would constitute evidence of NP. Although to date there are still no measurements on $K_{L, S} \rightarrow \notin$ , from the available data [22] on the visible decay modes of $K_{L, S}$ one can extract indirect upper bounds on their invisible branching fractions [33]:

$$
\mathcal{B}\left(K_{L} \rightarrow \not E\right)<6.3 \times 10^{-4}, \quad \mathcal{B}\left(K_{S} \rightarrow \not ̈\right)<1.1 \times 10^{-4}
$$

both at $95 \%$ CL. Hence there is still plenty of room for NP to influence these decays, particularly via the couplings $\tilde{\mathrm{C}}_{f}^{\mathrm{A}, \mathrm{S}, \mathrm{P}}$, as eq. (4.6) indicates.

For the $K \rightarrow \pi \nu \bar{\nu}$ modes, the SM predictions are $\mathcal{B}\left(K^{+} \rightarrow \pi^{+} \nu \nu\right)_{\mathrm{SM}}=\left(8.5_{-1.2}^{+1.0}\right) \times$ $10^{-11}$ and $\mathcal{B}\left(K_{L} \rightarrow \pi^{0} \nu \bar{\nu}\right)_{\mathrm{SM}}=\left(3.2_{-0.7}^{+1.1}\right) \times 10^{-11}$ [34]. These are not very far from their measurements $\mathcal{B}\left(K^{+} \rightarrow \pi^{+} \nu \nu\right)_{\exp }=1.7(1.1) \times 10^{-10}[22]$ and $\mathcal{B}\left(K_{L} \rightarrow \pi^{0} \nu \bar{\nu}\right)_{\exp }<3.0 \times$ $10^{-9}$ at $90 \%$ CL [21]. It follows that the effects of NP on these modes, and consequently its contributions to $\mathrm{C}_{f}^{\mathrm{V}, \mathrm{A}, \mathrm{S}, \mathrm{P}}$, expectedly cannot be considerable.

As regards the four-body kaon decays, the SM expectations are $\mathcal{B}\left(K^{-} \rightarrow \pi^{0} \pi^{-} \nu \bar{\nu}\right)_{\mathrm{SM}} \sim$ $10^{-14}$ and $\mathcal{B}\left(K_{L} \rightarrow \pi^{0} \pi^{0} \nu \bar{\nu}\right)_{\mathrm{SM}} \sim 10^{-13}[13,35,36]$. These are way below the existing empirical bounds $\mathcal{B}\left(K^{-} \rightarrow \pi^{0} \pi^{-} \nu \bar{\nu}\right)_{\exp }<4.3 \times 10^{-5}[37]^{6}$ and $\mathcal{B}\left(K_{L} \rightarrow \pi^{0} \pi^{0} \nu \bar{\nu}\right)_{\exp }<$ $8.1 \times 10^{-7}[38]$, both at $90 \% \mathrm{CL}$. We may then impose

$$
\mathcal{B}\left(K^{-} \rightarrow \pi^{0} \pi^{-} \mathbb{E}\right)<4 \times 10^{-5}, \quad \mathcal{B}\left(K_{L} \rightarrow \pi^{0} \pi^{0} \notin\right)<8 \times 10^{-7},
$$

which imply further restraints on $\tilde{\mathrm{C}}_{f}^{\mathrm{V}, \mathrm{A}, \mathrm{S}, \mathrm{P}}$.

\subsection{Beyond SM}

As mentioned in the preceding subsection, the current $K \rightarrow \pi \nu \bar{\nu}$ data do not leave ample room for NP to affect $C_{f}^{\mathrm{V}, \mathrm{A}, \mathrm{S}, \mathrm{P}}$ greatly. More specifically, our numerical scans reveal that the allowed values of these couplings alone cannot produce $\mathcal{B}\left(\mathfrak{B} \rightarrow \mathfrak{B}^{\prime} f \bar{f}\right)$ above $10^{-11}$, and so this scenario would be out of BESIII reach according to table 2 .

\footnotetext{
${ }^{6}$ The search region was defined by $90 \mathrm{MeV}<P_{\pi^{-}}<188 \mathrm{MeV}$ and $135 \mathrm{MeV}<E_{\pi^{0}}<180 \mathrm{MeV}$ [37].
} 
Therefore, hereafter we concentrate on the possibility that NP can generate sizable effects only via $\tilde{c}_{f}^{\mathrm{V}, \mathrm{A}, \mathrm{S}, \mathrm{P}}$. For simplicity, we assume that $f$ is a nonstandard fermion which is sufficiently light compared to the mass difference between the initial and final baryons, so that we can approximately set $m_{f}$ to zero in numerical work. It follows that only the constraints on $K \rightarrow f \bar{f}$ and $K \rightarrow \pi \pi^{\prime} f \bar{f}$ need to be addressed when dealing with the hyperon decays. Moreover, since the NP contributions do not interfere with $s \rightarrow d \nu \bar{\nu}$, the tiny SM contributions to these processes with missing energy can be ignored.

Integrating the differential rates of the baryon decays, for $m_{f}=0$ we arrive at the branching fractions

$$
\begin{aligned}
\mathcal{B}(\Lambda \rightarrow n f \bar{f})= & 4.4\left(\left|\mathrm{C}_{f}^{\mathrm{V}}\right|^{2}+\left|\mathrm{C}_{f}^{\mathrm{A}}\right|^{2}\right)+11\left(\left|\mathrm{C}_{f}^{\mathrm{S}}\right|^{2}+\left|\mathrm{C}_{f}^{\mathrm{P}}\right|^{2}\right) \\
& \left.+7.0\left(\left|\tilde{\mathrm{c}}_{f}^{\mathrm{V}}\right|^{2}+\left|\tilde{\mathrm{c}}_{f}^{\mathrm{A}}\right|^{2}\right)+2.3\left(\left|\tilde{\mathrm{c}}_{f}^{\mathrm{S}}\right|^{2}+\left|\tilde{\mathrm{c}}_{f}^{\mathrm{P}}\right|^{2}\right)\right] 10^{7} \mathrm{GeV}^{4}, \\
\mathcal{B}\left(\Sigma^{+} \rightarrow p f \bar{f}\right)= & {\left[5.1\left(\left|\mathrm{C}_{f}^{\mathrm{V}}\right|^{2}+\left|\mathrm{C}_{f}^{\mathrm{A}}\right|^{2}\right)+26\left(\left|\mathrm{C}_{f}^{\mathrm{S}}\right|^{2}+\left|\mathrm{C}_{f}^{\mathrm{P}}\right|^{2}\right)\right.} \\
& \left.+1.8\left(\left|\tilde{\mathrm{c}}_{f}^{\mathrm{V}}\right|^{2}+\left|\tilde{\mathrm{c}}_{f}^{\mathrm{A}}\right|^{2}\right)+1.4\left(\left|\tilde{\mathrm{c}}_{f}^{\mathrm{S}}\right|^{2}+\left|\tilde{\mathrm{c}}_{f}^{\mathrm{P}}\right|^{2}\right)\right] 10^{7} \mathrm{GeV}^{4}, \\
\mathcal{B}\left(\Xi^{0} \rightarrow \Lambda f \bar{f}\right)= & {\left[9.3\left(\left|\mathrm{C}_{f}^{\mathrm{V}}\right|^{2}+\left|\mathrm{C}_{f}^{\mathrm{A}}\right|^{2}\right)+30\left(\left|\mathrm{C}_{f}^{\mathrm{S}}\right|^{2}+\left|\mathrm{C}_{f}^{\mathrm{P}}\right|^{2}\right)\right.} \\
& \left.+1.0\left(\left|\tilde{\mathrm{c}}_{f}^{\mathrm{V}}\right|^{2}+\left|\tilde{\mathrm{c}}_{f}^{\mathrm{A}}\right|^{2}\right)+0.4\left(\left|\tilde{\mathrm{c}}_{f}^{\mathrm{S}}\right|^{2}+\left|\tilde{\mathrm{c}}_{f}^{\mathrm{P}}\right|^{2}\right)\right] 10^{7} \mathrm{GeV}^{4}, \\
\mathcal{B}\left(\Xi^{0} \rightarrow \Sigma^{0} f \bar{f}\right)= & 0.29\left(\left|\mathrm{C}_{f}^{\mathrm{V}}\right|^{2}+\left|\mathrm{C}_{f}^{\mathrm{A}}\right|^{2}\right)+0.34\left(\left|\mathrm{C}_{f}^{\mathrm{S}}\right|^{2}+\left|\mathrm{C}_{f}^{\mathrm{P}}\right|^{2}\right) \\
& \left.+1.4\left(\left|\tilde{\mathrm{c}}_{f}^{\mathrm{V}}\right|^{2}+\left|\tilde{\mathrm{c}}_{f}^{\mathrm{A}}\right|^{2}\right)+0.20\left(\left|\tilde{\mathrm{c}}_{f}^{\mathrm{S}}\right|^{2}+\left|\tilde{\mathrm{c}}_{f}^{\mathrm{P}}\right|^{2}\right)\right] 10^{7} \mathrm{GeV}^{4}, \\
\mathcal{B}\left(\Xi^{-} \rightarrow \Sigma^{-} f \bar{f}\right)= & {\left[0.35\left(\left|\mathrm{C}_{f}^{\mathrm{V}}\right|^{2}+\left|\mathrm{C}_{f}^{\mathrm{A}}\right|^{2}\right)+0.42\left(\left|\mathrm{C}_{f}^{\mathrm{S}}\right|^{2}+\left|\mathrm{C}_{f}^{\mathrm{P}}\right|^{2}\right)\right.} \\
& \left.+1.7\left(\left|\tilde{\mathrm{c}}_{f}^{\mathrm{V}}\right|^{2}+\left|\tilde{\mathrm{c}}_{f}^{\mathrm{A}}\right|^{2}\right)+0.25\left(\left|\tilde{\mathrm{c}}_{f}^{\mathrm{S}}\right|^{2}+\left|\tilde{\mathrm{c}}_{f}^{\mathrm{P}}\right|^{2}\right)\right] 10^{7} \mathrm{GeV}^{4}, \\
\mathcal{B}\left(\Omega^{-} \rightarrow \Xi^{-} f \bar{f}\right)= & {\left[7.9\left(\left|\tilde{\mathrm{c}}_{f}^{\mathrm{V}}\right|^{2}+\left|\tilde{\mathrm{c}}_{f}^{\mathrm{A}}\right|^{2}\right)+14\left(\left|\tilde{\mathrm{c}}_{f}^{\mathrm{S}}\right|^{2}+\left|\tilde{\mathrm{c}}_{f}^{\mathrm{P}}\right|^{2}\right)\right] 10^{8} \mathrm{GeV}^{4} . }
\end{aligned}
$$

All these results have incorporated the form factors mentioned in section 3 . In the kaon sector, with $m_{f}=0$, for the two-body decays we get

leading to

$$
\begin{aligned}
& \mathcal{B}\left(K_{L} \rightarrow f \bar{f}\right)=2.9\left[\left(\operatorname{Im} \tilde{c}_{f}^{\mathrm{S}}\right)^{2}+\left(\operatorname{Re} \tilde{c}_{f}^{\mathrm{P}}\right)^{2}\right] 10^{14} \mathrm{GeV}^{4}, \\
& \mathcal{B}\left(K_{S} \rightarrow f \bar{f}\right)=5.1\left[\left(\operatorname{Re} \tilde{c}_{f}^{\mathrm{S}}\right)^{2}+\left(\operatorname{Im} \tilde{c}_{f}^{\mathrm{P}}\right)^{2}\right] 10^{11} \mathrm{GeV}^{4},
\end{aligned}
$$

$$
\left|\tilde{\mathrm{c}}_{f}^{\mathrm{S}}\right|^{2}+\left|\tilde{\mathrm{c}}_{f}^{\mathrm{P}}\right|^{2}=\frac{3.4 \times 10^{-15}}{\mathrm{GeV}^{4}} \mathcal{B}\left(K_{L} \rightarrow f \bar{f}\right)+\frac{2.0 \times 10^{-12}}{\mathrm{GeV}^{4}} \mathcal{B}\left(K_{S} \rightarrow f \bar{f}\right),
$$

and for the four-body decays

$$
\begin{aligned}
\mathcal{B}\left(K^{-} \rightarrow \pi^{-} \pi^{0} f \bar{f}\right) & =\left[6.3\left(\left|\tilde{\mathrm{c}}_{f}^{\mathrm{V}}\right|^{2}+\left|\tilde{\mathrm{c}}_{f}^{\mathrm{A}}\right|^{2}\right)+2.0\left(\left|\tilde{\mathrm{c}}_{f}^{\mathrm{S}}\right|^{2}+\left|\tilde{\mathrm{c}}_{f}^{\mathrm{P}}\right|^{2}\right)\right] 10^{5} \mathrm{GeV}^{4}, \\
\mathcal{B}\left(K_{L} \rightarrow \pi^{0} \pi^{0} f \bar{f}\right) & =\left[8.5\left(\operatorname{Re} \tilde{\mathrm{c}}_{f}^{\mathrm{V}}\right)^{2}+8.5\left(\operatorname{Re} \tilde{\mathrm{c}}_{f}^{\mathrm{A}}\right)^{2}+16\left(\operatorname{Im} \tilde{\mathrm{c}}_{f}^{\mathrm{S}}\right)^{2}+16\left(\operatorname{Re} \tilde{\mathrm{c}}_{f}^{\mathrm{P}}\right)^{2}\right] 10^{6} \mathrm{GeV}^{4} .
\end{aligned}
$$

Evidently, all the interference terms with different couplings have vanished as $m_{f} \rightarrow 0$. 
We can now look at a couple of representative instances with different choices of nonvanishing couplings, which we take to be all real to ignore any new source of $C P$ violation. If only $\tilde{\mathrm{C}}_{f}^{\mathrm{S}, \mathrm{P}}$ are nonzero, we find that the $K \rightarrow \notin$ restrictions in eq. (5.2) are more stringent than the $K \rightarrow \pi \pi^{\prime} \mathbb{E}$ ones in eq. (5.3) and, with the aid of eq. (5.9), lead to $\left|\tilde{\mathrm{c}}_{f}^{\mathrm{S}}\right|^{2}+\left|\tilde{\mathrm{c}}_{f}^{\mathrm{P}}\right|^{2}<2.2 \times 10^{-16} \mathrm{GeV}^{-4}$. Combining this with eqs. (5.4)-(5.7), we obtain

$$
\begin{aligned}
\mathcal{B}(\Lambda \rightarrow n f \bar{f}) & <5.0 \times 10^{-9}, & \mathcal{B}\left(\Sigma^{+} \rightarrow p f \bar{f}\right) & <3.0 \times 10^{-9}, \\
\mathcal{B}\left(\Xi^{0} \rightarrow \Lambda f \bar{f}\right) & <9.3 \times 10^{-10}, & \mathcal{B}\left(\Omega^{-} \rightarrow \Xi^{-} f \bar{f}\right) & <3.0 \times 10^{-7},
\end{aligned}
$$

and smaller numbers for $\mathcal{B}\left(\Xi^{0,-} \rightarrow \Sigma^{0,-} f \bar{f}\right)$. The upper ends of these ranges far exceed the respective SM values quoted in table 2 but are still roughly two orders of magnitude beyond the expected BESIII reach.

If only $\tilde{\mathrm{c}}_{f}^{\mathrm{V}, \mathrm{A}}$ are nonzero, then according to eq. (5.8) the $K \rightarrow \not$ constraints no longer apply as $\tilde{\mathrm{c}}_{f}^{\mathrm{V}, \mathrm{A}}$ do not affect these decays in the $m_{f}=0$ limit, but the $K \rightarrow \pi \pi^{\prime} \notin$ bounds still matter, the $K_{L} \rightarrow \pi^{0} \pi^{0} \not$ one being the stronger and yielding $\left(\operatorname{Re} \tilde{c}_{f}^{\mathrm{V}}\right)^{2}+\left(\operatorname{Re} \tilde{\mathrm{c}}_{f}^{\mathrm{A}}\right)^{2}<$ $9.4 \times 10^{-14} \mathrm{GeV}^{-4}$. This now translates into

$$
\begin{aligned}
\mathcal{B}(\Lambda \rightarrow n f \bar{f}) & <6.6 \times 10^{-6}, & \mathcal{B}\left(\Sigma^{+} \rightarrow p f \bar{f}\right) & <1.7 \times 10^{-6}, \\
\mathcal{B}\left(\Xi^{0} \rightarrow \Lambda f \bar{f}\right) & <9.4 \times 10^{-7}, & \mathcal{B}\left(\Xi^{0} \rightarrow \Sigma^{0} f \bar{f}\right) & <1.3 \times 10^{-6}, \\
\mathcal{B}\left(\Xi^{-} \rightarrow \Sigma^{-} f \bar{f}\right) & <1.6 \times 10^{-6}, & \mathcal{B}\left(\Omega^{-} \rightarrow \Xi^{-} f \bar{f}\right) & <7.5 \times 10^{-5},
\end{aligned}
$$

most of which have upper values exceeding the corresponding estimated BESIII sensitivity levels quoted in table 2. This suggests that BESIII might discover NP hints in these processes or, if not, come up with improved restrictions on $\tilde{c}_{f}^{\mathrm{V}, \mathrm{A}}$.

If we let $\operatorname{Im} \tilde{c}_{f}^{V, A} \neq 0$, bigger branching fractions than those in eq. (5.12) could be achieved with purely imaginary $\tilde{\mathrm{c}}_{f}^{\mathrm{V}, \mathrm{A}}$, as they would escape the $K_{L} \rightarrow \pi^{0} \pi^{0} \notin$ restraint and be subject only to the weaker $K^{-} \rightarrow \pi^{0} \pi^{-\not}$ one, implying the mild limit $\left(\operatorname{Im} \tilde{c}_{f}^{V}\right)^{2}+$ $\left(\operatorname{Im} \tilde{c}_{f}^{A}\right)^{2}<6.4 \times 10^{-11} \mathrm{GeV}^{-4}$. This serves to indicate further the benefit of measuring these hyperon decays, which may test some of the NP couplings more stringently than the kaon decays.

\section{Conclusions}

We have explored the possibility that new physics contributes to the strangeness-changing transition $s \rightarrow d \mathbb{E}$, with missing energy in the final state. Depending on the sizes of the NP couplings involved and the masses of the emitted invisible particles, various changes could occur to the SM predictions for rare kaon and hyperon $|\Delta S|=1$ decays with missing energy. We have learned that the current data on $K \rightarrow \pi \nu \bar{\nu}$ do not allow NP to influence the hyperon decays considerably if the underlying operators have mostly parity-even quark portions. On the other hand, if the NP operators have predominantly parity-odd, especially axial-vector, quark parts, the restraints implied by the $K \rightarrow$ invisible and $K \rightarrow \pi \pi^{\prime} \nu \bar{\nu}$ data are comparatively weaker. We have demonstrated that NP with the latter kind of interactions could cause the hyperon rates to be substantially amplified with respect to their SM expectations and have large values potentially testable in the ongoing BESIII experiment. This well illustrates that these rare hyperon decays and their kaon counterparts are complementary to each other as probes of possible NP in $s \rightarrow d \mathbb{E}$. 


\section{Acknowledgments}

This research was supported in part by the MOE Academic Excellence Program (Grant No. 105R891505).

\section{A Correspondences between quark and hadron transitions}

From the chiral Lagrangian that is at leading order in the derivative and $s$-quark-mass $\left(m_{s}\right)$ expansions and describes the strong interactions among the lightest octet baryons and mesons and decuplet baryons [39-41], one can extract correspondences between quark densities or currents and hadronic transitions [42]. From the results of ref. [42] pertaining to the $|\Delta S|=1$ processes under discussion, we can infer

$$
\begin{aligned}
\bar{d} \gamma_{\eta} s \Leftrightarrow & -\sqrt{\frac{3}{2}} \bar{n} \gamma_{\eta} \Lambda-\bar{p} \gamma_{\eta} \Sigma^{+}+\sqrt{\frac{3}{2}} \bar{\Lambda} \gamma_{\eta} \Xi^{0}-\frac{1}{\sqrt{2}} \overline{\Sigma^{0}} \gamma_{\eta} \Xi^{0}+\overline{\Sigma^{-}} \gamma_{\eta} \Xi^{-} \\
& +i\left(\pi^{+} \partial_{\eta} K^{-}-K^{-} \partial_{\eta} \pi^{+}\right)-\frac{i}{\sqrt{2}}\left(\pi^{0} \partial_{\eta} \bar{K}^{0}-\bar{K}^{0} \partial_{\eta} \pi^{0}\right)+\cdots \\
\bar{d} s \Leftrightarrow & \sqrt{\frac{3}{2}} \frac{m_{\Lambda}-m_{N}}{\hat{m}-m_{s}} \bar{n} \Lambda+\frac{m_{\Sigma}-m_{N}}{\hat{m}-m_{s}} \bar{p} \Sigma^{+}+\sqrt{\frac{3}{2}} \frac{m_{\Xi}-m_{\Lambda}}{m_{s}-\hat{m} \Xi^{0}} \\
& +\frac{m_{\Xi}-m_{\Sigma}}{\hat{m}-m_{s}}\left(\frac{\overline{\Sigma^{0}} \Xi^{0}}{\sqrt{2}}-\overline{\Sigma^{-}} \Xi^{-}\right)+B_{0}\left(\pi^{+} K^{-}-\frac{\pi^{0} \bar{K}^{0}}{\sqrt{2}}\right)+\cdots \\
\bar{d} \gamma_{\eta} \gamma_{5} s \Leftrightarrow & \frac{-D-3 F}{\sqrt{6}} \bar{n} \gamma_{\eta} \gamma_{5} \Lambda+(D-F) \bar{p} \gamma_{\eta} \gamma_{5} \Sigma^{+}-\frac{D-3 F}{\sqrt{6}} \bar{\Lambda} \gamma_{\eta} \gamma_{5} \Xi^{0} \\
& -\frac{D+F}{\sqrt{2}} \overline{\Sigma^{0}} \gamma_{\eta} \gamma_{5} \Xi^{0}+(D+F) \bar{\Sigma}^{-} \gamma_{\eta} \gamma_{5} \Xi^{-}+\mathcal{C} \Xi^{-} \Omega_{\eta}^{-} \\
& +\sqrt{2} f \partial_{\eta} \bar{K}^{0}+\frac{\pi^{+} \partial_{\eta} \pi^{0}-\pi^{0} \partial_{\eta} \pi^{+}}{f} K^{-}-\frac{\pi^{0} \partial_{\eta} \bar{K}^{0}-\overline{K^{0}} \partial_{\eta} \pi^{0}}{3 \sqrt{2} f} \pi^{0}+\cdots \\
\bar{d} \gamma_{5} s \Leftrightarrow & i \sqrt{2} B_{0} f \bar{K}^{0}-\frac{i B_{0} \pi^{0} \pi^{0} \bar{K}^{0}}{3 \sqrt{2} f}+\cdots,
\end{aligned}
$$

where $m_{N, \Sigma, \Xi}$ are isospin-averaged masses of the nucleons, $\Sigma^{ \pm, 0}$, and $\Xi^{0,-}$, respectively, $\hat{m}$ is the average mass of the $u$ and $d$ quarks, $B_{0}=m_{K}^{2} /\left(\hat{m}+m_{s}\right)$, with $m_{K}$ being the average mass of $K^{0}$ and $K^{-}$, the free parameters $D, F$, and $\mathcal{C}$ occur in the lowest-order chiral Lagrangian and can be fixed from baryon decay data, $f=f_{K} / \sqrt{2}$, and the ellipses stand for terms not relevant to our analysis.

At the same order in the chiral expansion, the baryonic matrix elements of $\bar{d}\left(\gamma^{\eta}, 1\right) \gamma_{5} s$ and $\left\langle\pi \pi^{\prime}\left|\bar{d}\left(\gamma^{\eta}, 1\right) \gamma_{5} s\right| \bar{K}\right\rangle$ also receive contributions from kaon-pole diagrams involving $\left\langle 0\left|\bar{d}\left(\gamma^{\eta}, 1\right) \gamma_{5} s\right| \bar{K}^{0}\right\rangle$ from eqs. (A.3) and (A.4) and strong vertices from the lowest-order strong chiral Lagrangian $\mathcal{L}_{\mathrm{s}}$. The pertinent terms are given by

$$
\begin{aligned}
\mathcal{L}_{\mathrm{s}} \supset & {\left[\frac{-D-3 F}{\sqrt{6}} \bar{n} \gamma_{\eta} \gamma_{5} \Lambda+(D-F) \bar{p} \gamma_{\eta} \gamma_{5} \Sigma^{+}-\frac{D-3 F}{\sqrt{6}} \bar{\Lambda} \gamma_{\eta} \gamma_{5} \Xi^{0}\right.} \\
& \left.-\frac{D+F}{\sqrt{2}} \overline{\Sigma^{0}} \gamma_{\eta} \gamma_{5} \Xi^{0}+(D+F) \overline{\Sigma^{-}} \gamma_{\eta} \gamma_{5} \Xi^{-}+\mathcal{C} \overline{\Xi^{-}} \Omega_{\eta}^{-}\right] \frac{\partial^{\eta} K^{0}}{\sqrt{2} f} \\
& +\frac{\pi^{0} \stackrel{\leftrightarrow}{\partial} \eta \pi^{+}}{\sqrt{8} f^{2}} K^{0} \stackrel{\leftrightarrow}{\partial}_{\eta} K^{-}+\frac{K^{0} \stackrel{\leftrightarrow}{\partial} \eta \pi^{0}}{12 f^{2}} \pi^{0} \stackrel{\leftrightarrow}{\partial}_{\eta} \bar{K}^{0}+\frac{m_{K}^{2}+m_{\pi}^{2}}{12 f^{2}} \pi^{0} \pi^{0} K^{0} \bar{K}^{0},
\end{aligned}
$$


where $X \stackrel{\leftrightarrow}{\partial}_{\eta} Y=X \partial_{\eta} Y-Y \partial_{\eta} X$. From this and the previous paragraphs, we arrive at the hadronic matrix elements in eqs. (3.1), (3.3), and (4.1)-(4.3), in the limit that $f_{+, 0}=1$.

In numerical work, we employ the observed hadron masses from ref. [22] and the lightquark mass values $\hat{m}=\left(m_{u}+m_{d}\right) / 2=4.4 \mathrm{MeV}$ and $m_{s}=120 \mathrm{MeV}$ at a renormalization scale of $1 \mathrm{GeV}$. Moreover, we adopt $D=0.81$ and $F=0.46$ determined from fitting to the data on hyperon semileptonic decays and $\mathcal{C}=1.7$ from the measurements of strong decays of the lightest decuplet spin-3/2 baryons into an octet spin- $1 / 2$ baryon and a pion [22].

\section{B Additional kaon decay formulas}

The decay amplitudes for $K_{L, S}$ are connected to those for $K^{0}$ and $\bar{K}^{0}$ through the approximate relations $\sqrt{2} K_{L, S}=K^{0} \pm \bar{K}^{0}$. Thus, for $K_{L, S} \rightarrow f \bar{f}$ induced by $\mathcal{L}_{f}$ in eq. (2.1), with eq. (4.1) we derive $S_{K_{L, S} f}$ and $P_{K_{L, S} f}$ in eq. (4.6).

For $K^{-} \rightarrow \pi^{-} f \bar{f}$ and $K_{L} \rightarrow \pi^{0} f \bar{f}$, from eqs. (2.1) and (4.2), we find the $S$ and $P$ terms in eq. (4.7) to be

$$
\begin{aligned}
S_{K^{-} \pi^{-}} & =2 f_{+} \not p_{K} \mathrm{C}_{f}^{\mathrm{V}}+B_{0} f_{0} \mathrm{C}_{f}^{\mathrm{S}}, \\
P_{K^{-} \pi^{-} f} & =2\left[\left(f_{-}-f_{+}\right) m_{f}+f_{+} \not \not_{K}\right] \mathrm{C}_{f}^{\mathrm{A}}+B_{0} f_{0} \mathrm{C}_{f}^{\mathrm{P}}, \\
S_{K_{L} \pi^{0} f} & =-2 i f_{+} \not p_{K} \operatorname{Im} \mathrm{C}_{f}^{\mathrm{V}}-B_{0} f_{0} \operatorname{Re}_{f}^{\mathrm{S}}, \\
P_{K_{L} \pi^{0} f} & =2 i\left[\left(f_{+}-f_{-}\right) m_{f}-f_{+} \not p_{K}\right] \operatorname{Im~C}_{f}^{\mathrm{A}}-i B_{0} f_{0} \operatorname{Im~C}_{f}^{\mathrm{P}} .
\end{aligned}
$$

For $K_{S} \rightarrow \pi^{0} f \bar{f}$, the $S$ and $P$ formulas are equal to $S_{K_{L} \pi^{0} f}$ and $P_{K_{L} \pi^{0} f}$ but with $\operatorname{Re}_{f}$ and $-i \operatorname{Im} C_{f}$ interchanged. The differential rate of the $K_{L}$ decay is then

$$
\begin{aligned}
\Gamma_{K_{L} \rightarrow \pi^{0} f \bar{f}}^{\prime}=\frac{\beta \lambda_{K^{0} \pi^{0}}^{1 / 2} f_{0}^{2}}{128 \pi^{3} m_{K^{0}}^{3}}\{ & \frac{3-\beta^{2}}{3 f_{0}^{2}} \lambda_{K^{0} \pi^{0}} f_{+}^{2}\left(\operatorname{Im~C} C_{f}^{\mathrm{V}}\right)^{2} \\
+ & {\left[\frac{2 \beta^{2} f_{+}^{2}}{3 f_{0}^{2}} \lambda_{K^{0} \pi^{0}}+\frac{4 m_{f}^{2}}{\hat{s}} \Delta_{K^{0} \pi^{0}}^{4}\right]\left(\operatorname{Im} C_{f}^{\mathrm{A}}\right)^{2} } \\
& \left.+4 B_{0} m_{f} \Delta_{K^{0} \pi^{0}}^{2} \operatorname{Im~C}_{f}^{\mathrm{A}} \operatorname{Im~C}_{f}^{\mathrm{P}}+B_{0}^{2}\left[\beta^{2}\left(\operatorname{Re}_{f}^{\mathrm{S}}\right)^{2}+\left(\operatorname{Im} C_{f}^{\mathrm{P}}\right)^{2}\right] \hat{s}\right\},
\end{aligned}
$$

where $\Delta_{K \pi}^{2}=m_{K}^{2}-m_{\pi}^{2}$. For $\Gamma_{K_{S} \rightarrow \pi^{0} f \bar{f}}^{\prime}$, the expression is obtainable from $\Gamma_{K_{L} \rightarrow \pi^{0} f \bar{f}}^{\prime}$ by interchanging $\operatorname{Re}_{f}$ and $\operatorname{Im~C}_{f}$, while $\Gamma_{K^{-} \rightarrow \pi^{-} f \bar{f}}^{\prime}$ is the same as $\Gamma_{K_{L} \rightarrow \pi^{0} f \bar{f}}^{\prime}+\Gamma_{K_{S} \rightarrow \pi^{0} f \bar{f}}^{\prime}$ but with $m_{K^{0}, \pi^{0}}$ replaced with $m_{K^{-}, \pi^{-}}$.

For the four-body decays, we have [13]

$$
\begin{gathered}
\frac{d^{2} \Gamma_{K^{-} \rightarrow \pi^{0} \pi^{-} f \bar{x}}}{d \hat{s} d \hat{\varsigma}}=\frac{\beta_{\hat{\zeta}} \tilde{\lambda}^{1 / 2} m_{K}}{1536 \pi^{5} f_{K}^{2}}\left[\mathrm{~F}_{1}\left|\tilde{\mathrm{c}}_{f}^{\mathrm{V}}\right|^{2}+\mathrm{F}_{1}^{\prime}\left|\tilde{\mathrm{c}}_{f}^{\mathrm{A}}\right|^{2}+\mathrm{F}_{2}\left|\tilde{\mathrm{c}}_{f}^{\mathrm{S}}\right|^{2}+\mathrm{F}_{2}^{\prime}\left|\tilde{\mathrm{c}}_{f}^{\mathrm{P}}\right|^{2}\right. \\
\left.\quad+\mathrm{F}_{12}\left(\operatorname{Re} \tilde{\mathrm{c}}_{f}^{\mathrm{A}} \operatorname{Re} \tilde{\mathrm{c}}_{f}^{\mathrm{P}}+\operatorname{Im} \tilde{\mathrm{c}}_{f}^{\mathrm{A}} \operatorname{Im} \tilde{\mathrm{c}}_{f}^{\mathrm{P}}\right)\right] \\
\frac{d^{2} \Gamma_{K_{L} \rightarrow \pi^{0} \pi^{0} f \bar{x}}}{d \hat{s} d \hat{\varsigma}}=\frac{\beta_{\hat{\zeta}} \tilde{\lambda}^{1 / 2} m_{K}}{3072 \pi^{5} f_{K}^{2}}\left(\mathrm{~F}_{3}\left|\operatorname{Re} \tilde{\mathrm{c}}_{f}^{\mathrm{V}}\right|^{2}+\mathrm{F}_{3}^{\prime}\left|\operatorname{Re} \tilde{\mathrm{c}}_{f}^{\mathrm{A}}\right|^{2}+\mathrm{F}_{4}\left|\operatorname{Im} \tilde{\mathrm{c}}_{f}^{\mathrm{S}}\right|^{2}+\mathrm{F}_{4}^{\prime}\left|\operatorname{Re} \tilde{\mathrm{c}}_{f}^{\mathrm{P}}\right|^{2}\right. \\
\left.+\mathrm{F}_{34} \operatorname{Re} \tilde{\mathrm{c}}_{f}^{\mathrm{A}} \operatorname{Re} \tilde{\mathrm{c}}_{f}^{\mathrm{P}}\right)
\end{gathered}
$$


where

$$
\begin{array}{rlrl}
\beta_{\hat{\varsigma}} & =\sqrt{1-\frac{4 m_{\pi}^{2}}{\hat{\varsigma}}}, & \hat{\varsigma} & =\left(p_{0}+p_{-}\right)^{2}=\left(p_{1}+p_{2}\right)^{2}, \\
\tilde{\lambda} & =m_{K}^{4}-2(\hat{s}+\hat{\varsigma}) m_{K}^{2}+(\hat{s}-\hat{\varsigma})^{2}, & & \mathrm{~F}_{1,3}^{\prime}=\beta^{2} \lambda_{1,3}+\lambda_{1,3}^{\prime} \frac{m_{f}^{2}}{\hat{s}}, \\
\mathrm{~F}_{1,3} & =\lambda_{1,3} \frac{2 m_{f}^{2}+\hat{s}}{\hat{s}}, & \mathrm{~F}_{2,4}^{\prime} & =\frac{\lambda_{1,3}^{\prime} B_{0}^{2} \hat{s}}{4 m_{K}^{4}}, \\
\mathrm{~F}_{2,4} & =\beta^{2} \mathrm{~F}_{2,4}^{\prime}, & \mathrm{F}_{34} & =-\lambda_{3}^{\prime} \frac{B_{0} m_{f}}{m_{K}^{2}} \\
\mathrm{~F}_{12} & =-\lambda_{1}^{\prime} \frac{B_{0} m_{f}}{m_{K}^{2}}, & \lambda_{3} & =\frac{\tilde{\lambda}}{4 m_{K}^{4}}, \\
\lambda_{1} & =\frac{\beta_{\hat{\varsigma}}^{2}}{m_{K}^{4}}\left(\hat{s} \hat{\varsigma}+\frac{\tilde{\lambda}}{12}\right), & \lambda_{3}^{\prime} & =\frac{12 \hat{s} \hat{\varsigma}+3 \tilde{\lambda}}{2\left(m_{K}^{2}-\hat{s}\right)^{2}} . \\
\lambda_{1}^{\prime} & =\frac{\beta_{\hat{\varsigma}}^{2} \tilde{\lambda}}{2\left(m_{K}^{2}-\hat{s}\right)^{2}}, &
\end{array}
$$

We compute the rates by integrating the double-differential rates in eq. (B.3) over the intervals $4 m_{f}^{2} \leq \hat{s} \leq\left(m_{K}-2 m_{\pi}\right)^{2}$ and $4 m_{\pi}^{2} \leq \hat{\varsigma} \leq\left(m_{K}-\hat{s}^{1 / 2}\right)^{2}$.

Open Access. This article is distributed under the terms of the Creative Commons Attribution License (CC-BY 4.0), which permits any use, distribution and reproduction in any medium, provided the original author(s) and source are credited.

\section{References}

[1] G. Buchalla, A.J. Buras and M.E. Lautenbacher, Weak decays beyond leading logarithms, Rev. Mod. Phys. 68 (1996) 1125 [hep-ph/9512380] [INSPIRE].

[2] W.J. Marciano and Z. Parsa, Rare kaon decays with "missing energy", Phys. Rev. D 53 (1996) R1.

[3] Y. Grossman and Y. Nir, $K(L) \rightarrow \pi^{0}$ neutrino anti-neutrino beyond the standard model, Phys. Lett. B 398 (1997) 163 [hep-ph/9701313] [inSPIRE].

[4] A.J. Buras, F. Schwab and S. Uhlig, Waiting for precise measurements of $K^{+} \rightarrow \pi^{+} \nu \bar{\nu}$ and $K_{L} \rightarrow \pi^{0} \nu \bar{\nu}$, Rev. Mod. Phys. 80 (2008) 965 [hep-ph/0405132] [INSPIRE].

[5] C.-J. Lee and J. Tandean, Minimal lepton flavor violation implications of the $b \rightarrow s$ anomalies, JHEP 08 (2015) 123 [arXiv: 1505. 04692] [INSPIRE].

[6] K. Fuyuto, W.-S. Hou and M. Kohda, $Z^{\prime}$-induced FCNC decays of top, beauty and strange quarks, Phys. Rev. D 93 (2016) 054021 [arXiv: 1512.09026] [INSPIRE].

[7] H.-B. Li, Prospects for rare and forbidden hyperon decays at BESIII, Front. Phys. (Beijing) 12 (2017) 121301 [arXiv: 1612.01775] [INSPIRE].

[8] C.-W. Chiang, X.-G. He, J. Tandean and X.-B. Yuan, $R_{K^{(*)}}$ and related $b \rightarrow s \ell \bar{\ell}$ anomalies in minimal flavor violation framework with $Z^{\prime}$ boson, Phys. Rev. D 96 (2017) 115022 [arXiv: 1706. 02696] [INSPIRE]. 
[9] X.-H. Hu and Z.-X. Zhao, Study of $s \rightarrow d \nu \bar{\nu}$ rare hyperon decays within the standard model and new physics, arXiv:1811.01478 [INSPIRE].

[10] X.-G. He and G. Valencia, $K^{+} \rightarrow \pi^{+} \nu \bar{\nu}$ and FCNC from non-universal $Z^{\prime}$ bosons, Phys. Rev. D 70 (2004) 053003 [hep-ph/0404229] [INSPIRE].

[11] C. Bird, R.V. Kowalewski and M. Pospelov, Dark matter pair-production in $b \rightarrow s$ transitions, Mod. Phys. Lett. A 21 (2006) 457 [hep-ph/0601090] [InSPIRE].

[12] X.-G. He, S.-Y. Ho, J. Tandean and H.-C. Tsai, Scalar dark matter and standard model with four generations, Phys. Rev. D 82 (2010) 035016 [arXiv: 1004.3464] [INSPIRE].

[13] J.F. Kamenik and C. Smith, FCNC portals to the dark sector, JHEP 03 (2012) 090 [arXiv: 1111.6402] [INSPIRE].

[14] M.J. Dolan, F. Kahlhoefer, C. McCabe and K. Schmidt-Hoberg, A taste of dark matter: Flavour constraints on pseudoscalar mediators, JHEP 03 (2015) 171 [Erratum ibid. 07 (2015) 103] [arXiv: 1412.5174] [INSPIRE].

[15] S.N. Gninenko and N.V. Krasnikov, Invisible $K_{L}$ decays as a probe of new physics, Phys. Rev. D 92 (2015) 034009 [arXiv: 1503.01595] [INSPIRE].

[16] A. Abada et al., Sterile neutrinos facing kaon physics experiments, Phys. Rev. D 95 (2017) 075023 [arXiv: 1612.04737] [INSPIRE].

[17] X.-G. He, G. Valencia and K. Wong, Constraints on new physics from $K \rightarrow \pi \nu \bar{\nu}$, Eur. Phys. J. C 78 (2018) 472 [arXiv:1804.07449] [InSPIRE].

[18] D. Barducci, M. Fabbrichesi and E. Gabrielli, Neutral hadrons disappearing into the darkness, Phys. Rev. D 98 (2018) 035049 [arXiv:1806.05678] [INSPIRE].

[19] S. Matsumoto, Y.-L.S. Tsai and P.-Y. Tseng, Light fermionic WIMP dark matter with light scalar mediator, arXiv:1811.03292 [INSPIRE].

[20] NA62 collaboration, First search for $K^{+} \rightarrow \pi^{+} \nu \bar{\nu}$ using the decay-in-flight technique, Phys. Lett. B 791 (2019) 156 [arXiv:1811.08508] [INSPIRE].

[21] KOTO collaboration, Search for the $K_{L} \rightarrow \pi^{0} \nu \bar{\nu}$ and $K_{L} \rightarrow \pi^{0} X^{0}$ decays at the J-PARC KOTO experiment, Phys. Rev. Lett. 122 (2019) 021802 [arXiv:1810.09655] [INSPIRE].

[22] Particle Data Group collaboration, Review of particle physics, Phys. Rev. D 98 (2018) 030001.

[23] M. Arteaga, E. Bertuzzo, C. Caniu Barros and Z. Tabrizi, Operators from flavored dark sectors running to low energy, Phys. Rev. D 99 (2019) 035022 [arXiv:1810.04747] [INSPIRE].

[24] P. Langacker, The physics of heavy $Z^{\prime}$ gauge bosons, Rev. Mod. Phys. 81 (2009) 1199 [arXiv: 0801.1345] [INSPIRE].

[25] A. Ismail, W.-Y. Keung, K.-H. Tsao and J. Unwin, Axial vector $Z^{\prime}$ and anomaly cancellation, Nucl. Phys. B 918 (2017) 220 [arXiv:1609.02188] [INSPIRE].

[26] A. Alves et al., Augury of darkness: the low-mass dark Z' portal, JHEP 04 (2017) 164 [arXiv:1612.07282] [INSPIRE].

[27] Bristol-Geneva-Heidelberg-Orsay-Rutherford-Strasbourg collaboration, Measurements of Hyperon Semileptonic Decays at the CERN super proton synchrotron. 1. The $\Sigma^{-} \rightarrow \Lambda e^{-}$anti-neutrino decay mode, Z. Phys. C 12 (1982) 307 [INSPIRE]. 
[28] S.Y. Hsueh et al., A high precision measurement of polarized $\sigma-\beta$ decay, Phys. Rev. D 38 (1988) 2056 [INSPIRE].

[29] J. Dworkin et al., High statistics measurement of $g(a) / g(v)$ in $\Lambda \rightarrow p+e^{-}+\bar{\nu}$, Phys. Rev. D 41 (1990) 780 [INSPIRE].

[30] NA48/I collaboration, Measurement of the branching ratios of the decays $\Xi_{0} \rightarrow \Sigma^{+} e^{-} \bar{\nu}_{e}$ and $\bar{\Xi}_{0} \rightarrow \bar{\Sigma}^{+} e^{+} \nu_{e}$, Phys. Lett. B 645 (2007) 36 [hep-ex/0612043] [INSPIRE].

[31] J. Charles et al., Current status of the standard model CKM fit and constraints on $\Delta F=2$ new physics, Phys. Rev. D 91 (2015) 073007 [arXiv:1501.05013] [INSPIRE].

[32] A.J. Buras, D. Buttazzo, J. Girrbach-Noe and R. Knegjens, $K^{+} \rightarrow \pi^{+} \nu \bar{\nu}$ and $K_{L} \rightarrow \pi^{0} \nu \bar{\nu}$ in the standard model: status and perspectives, JHEP 11 (2015) 033 [arXiv:1503.02693] [INSPIRE].

[33] S.N. Gninenko, Search for invisible decays of $\pi^{0}, \eta, \eta^{\prime}, K_{S}$ and $K_{L}$ : a probe of new physics and tests using the Bell-Steinberger relation, Phys. Rev. D 91 (2015) 015004 [arXiv: 1409.2288] [INSPIRE].

[34] C. Bobeth and A.J. Buras, Leptoquarks meet $\varepsilon^{\prime} / \varepsilon$ and rare Kaon processes, JHEP 02 (2018) 101 [arXiv: 1712.01295] [INSPIRE].

[35] L.S. Littenberg and G. Valencia, The decays $K \rightarrow \pi \pi$ neutrino anti-neutrino within the standard model, Phys. Lett. B 385 (1996) 379 [hep-ph/9512413] [INSPIRE].

[36] C.-W. Chiang and F.J. Gilman, $K(L, S) \rightarrow \pi \pi \nu \bar{\nu}$ decays within and beyond the standard model, Phys. Rev. D 62 (2000) 094026 [hep-ph/0007063] [INSPIRE].

[37] E787 collaboration, Search for the decay $K^{+} \rightarrow \pi^{+} \pi^{0} \nu \bar{\nu}$, Phys. Rev. D 63 (2001) 032004 [hep-ex/0009055] [INSPIRE].

[38] E391A collaboration, Study of the $K_{L}^{0} \rightarrow \pi^{0} \pi^{0} \nu \bar{\nu}$ decay, Phys. Rev. D 84 (2011) 052009 [arXiv: 1106.3404] [INSPIRE].

[39] J. Gasser and H. Leutwyler, Chiral perturbation theory to one loop, Annals Phys. 158 (1984) 142 [INSPIRE].

[40] J. Bijnens, H. Sonoda and M.B. Wise, On the validity of chiral perturbation theory for weak hyperon decays, Nucl. Phys. B 261 (1985) 185 [INSPIRE].

[41] E.E. Jenkins and A.V. Manohar, Chiral corrections to the baryon axial currents, Phys. Lett. B 259 (1991) 353 [INSPIRE].

[42] X.-G. He, J. Tandean and G. Valencia, Implications of a new particle from the hyperCP data on $\Sigma^{+} \rightarrow p \mu^{+} \mu^{-}$, Phys. Lett. B 631 (2005) 100 [hep-ph/0509041] [INSPIRE]. 